\title{
Speech Act Immigration in Korean Textbook for Social Integration Program
}

\section{사회통합 프로그램 한국어 교재에 나타난 한국어 화행 연구}

Jung-mi Jeon ${ }^{1}$

전정미 ${ }^{1}$

${ }^{1}$ Professor, Korean Language \& Litereture Department, Sang Myung University, S. Korea, meejeon@smu.ac.kr

\begin{abstract}
The purpose of this study is to examine the themes and functions selected as the contents of Korean language textbooks of the Social Integration Program from the perspective of speech act education, and to seek ways to improve the general Korean communication skills of immigrants. In order to analyze the textbook content and to identify its intended outcome, the performance review of the published textbook was examined, and in particular, the topics and functions were analyzed. As a result, positive elements have been found in the newly developed beginner and intermediate textbooks in terms of topics that can help migrants adjust to Korean society. However, insufficient course materials to support the themes especially in language functions and practical speech acts in diverse cultural contexts have been identified. In particular, there is a lack of opportunity to practice on how to continue a conversation along the different level, and cultural speech acts such as compliment, apology, refusal, and gratitude were not implemented. Education on cultural speech acts, which can be the basis for the immigrants to adapt to a new culture and engage in the society, is an essential part of Social Integration Programs, and therefore the education programs to strengthen this capacity should be supplemented.
\end{abstract}

Keywords: Korea Immigration \& Integration Program, Speech Act, Communicative Competence, Korean Textbook

요약: 이 연구의 목적은 사회통합 프로그램의 한국어 교재 내용으로 선정된 주제와 기능을 화행 교육의 관점에서 살펴보고, 이민자의 의사소통 능력 향상을 위한 방안을 모색하는 것이다. 교재 내용과 발간 의도를 확인하기 위해 교재 발간 결과 보고서를 살펴보았고, 특히 주제와 기능에 관한 부분을 분석하였다. 그 결과 새로 개발된 초급, 중급 교재에서는 이주민의 한국 사회 적응에 도움이 될만한 주제에 대해서 대해 다루고 있는 점은 매우 긍정적이다. 그러나 이를 뒷받침할 실제적 기능과 문화적 화행에 대한 제시가 부족하다는 점은 아쉬운 부분이다. 특히 난이도에 따라 대화 진행 방법에 대한 연습 기회가 부족하고, 칭찬, 사과, 거절, 감사와 같은 문화적 화행이 제시되지 않았다. 문화적 화행에 대한 교육은 이주민의 문화 적응과 더불어 의사소통의 참여자로서의 역할을 충분히 수행할 수 있도록 성장하고 발전할 수 있는 토대가 될 수 있는 필수적인

Received: March 23, 2021; 1st Review Result: May 07, 2021; 2nd Review Result: June 19, 2021 Accepted: July 31, 2021 
요소이기 때문에 이에 대한 보완이 필요하다.

핵심어: 사회통합 프로그램, 화행, 의사소통 능력, 한국어 교재

\section{1. 서론}

이 연구의 목적은 대한민국의 사회통합 프로그램 운영 방식과 사회통합 프로그램의 한국어 교재 '한국어와 한국문화'에 나타난 주제와 기능을 살펴보고, 이를 바탕으로 학습자들이 문화적 차이를 극복하고 한국 사회의 구성원으로서 원활한 의사소통 능력을 갖추는 데에 필요한 화행 교육의 방안을 모색하는 것이다.

한국 사회는 결혼이민자의 꾸준한 증가, 저출산과 고령화로 인한 외국 노동력의 유입, 유학생의 증가 등으로 국내 체류 외국인의 수가 2015년 대비 $32.9 \%(625,137$ 명)가 증가하였고, 2020년 7월 발간된 법무부 통계자료에 따르면, 2019년 12월 31일을 기준으로 인구 대비 체류 외국인은 2015년 3.69\%에서, 2019년 4.87\%로 나타나, 2,524,656명에 달한다. 이와 같은 변화는 우리 사회가 다양성과 이질성이 혼재하는 다문화 사회로 변모하고 있음을 시사한다. 따라서 문화적 차이에 의해 발생할 수도 있는 갈등 해소를 위해 의사소통의 중요성은 더욱 강조될 수밖에 없다[1]. 한국에 체류하고 있는 외국인이 문화적 차이에 의한 갈등을 줄이고, 한국 사회의 구성원으로서 생활하는 데 필요한 한국어와 경제, 사회, 문화 등 기본적인 소양을 체계적으로 익힐 수 있도록 법무부에서는 사회통합 프로그램(Korea Immigration \& integration program, KIIP)을 마련하여 운영하고 있다.

사회통합 프로그램과 관련된 선행 연구는, 프로그램 운영의 문제점 및 개선 방안에 대한 연구와 사회통합 프로그램 한국어 교육에서의 교육 내용에 관한 연구가 주를 이루고 있다. 한국어 교육의 관점에서 사회통합 프로그램의 교육과정과 교재, 평가 등에 대해 살펴본 연구로는 조항록(2012)을 시작으로[2] 한국어 교재에 사용된 대화문과 어휘, 문법적인 요소, 의사소통이 이루어지는 상황[3], 문화교육에서 다루는 내용과 같이 교재의 내용에 대한 연구[1][4], 교재의 구성 체계와 내용[5], 교육과정의 발전 단계와 교육과정 및 개편 교재[6], 문화교육의 내용과 목표 달성을 위한 내용의 타당도 검토, 교재 개선을 위한 방향[7] 등 사회통합 프로그램의 한국어 교육과정 전반에 대한 연구가 진행되었다. 이상의 선행 연구 결과를 종합하면, 사회통합 프로그램은 국가가 정책적으로 기획하여 운용하는 프로그램이기 때문에 교육의 목적과 방향은 분명하지만 다양한 의사소통 상황을 충분히 반영하는 교육 내용을 담고 있지 못한 부분이 있다고 지적한다.

사회통합 프로그램의 한국어 교재는 2013년도부터 사용되어 오던 것이 있었는데, 변화하는 시대 상황을 반영하기 위해 국립국어원의 용역으로 2020년 '한국어와 한국문화' 신규 교재와 이민자 유형별 보조자료가 개발되었다. 이에 이 연구에서는 사회통합 프로그램의 한국어 신규 교재 발간에 관한 1차년도 연구 보고서[8]의 내용을 토대로 한국어 의사소통 상황의 특징이 교재에 얼마나 적극적으로 반영되어 있는지 화행 교육의 관점에서 살펴보고자 한다. 사회통합 프로그램의 한국어 교재 개발에 관한 보고서는 교재 개발을 위한 기초 연구 보고서를 시작으로 2020년 1차 보고서, 2021년 2차 결과보고서가 발간되었다. 이 연구에서는 교재 집필의 의도와 방향 등이 상세히 제시되어 있는 1 차 보고서를 대상으로 살펴보았다. 먼저 한국어 초급과 중급 교재에서 
선정된 주제와 기능을 중심으로 화행 항목의 반영 정도를 중심으로 분석하고자 한다. 그리고 이를 토대로 맥락에 적합한 화행 수행 능력을 향상할 수 있는 방안에 대해 제안하고자 한다. 신규로 개발된 사회통합 프로그램의 한국어 교재에 나타난 의사소통 상황을 화행의 관점에서 살펴보는 것은 이민자들의 원활한 의사소통능력 함양에 필요한 요소가 무엇인지를 살펴보는 작업으로 매우 의미 있는 일이라 본다.

\section{2. 의사소통 능력과 화행 사용}

\section{1 화행의 개념과 유형}

화행 이론(Speech acts theory)은 발화가 행위를 수반한다는 기본적인 개념을 제안한 Austin(1962)에서 시작하여[9] Searle(1969)에 의해 체계화되었다[10]. 화행 이론의 중심적인 생각은 인간의 발화가 단순히 말의 차원에서 머무는 것이 아니라 사회 제도와 관습과 같 은 틀 안에서 이루어지는 하나의 행위라는 것이다. Austin(1962/1975: 94)에서는 언어로 행 위를 나타낸다는 말을 토대로 의사소통 상황에서 나타나는 발화를 화행이라고 규정하였 다. 어떤 말을 하는 것은 어떤 것을 하는 것(to say something is to do something)이고, 심지어 무엇인가를 말함으로써 무엇을 하는 것(even by saying something we do something)이라고 보 았다[9]. 사람들이 말하는 것은 여러 행동과 마찬가지로 행동의 하나라는 관점이다. 화행 이론에서는 모든 발화에는 화자의 의도가 포함되어 있을 뿐만 아니라 의사소통 상황에서 힘을 발휘하여 누군가에게 특정한 행위를 하게 만드는, 인간관계에 영향을 끼치는 역동 적인 행위라고 보았다. 그래서 우리가 언어를 사용할 때는 다음과 같은 세 가지 행위를 수반한다고 본다.

(1) 화행
a. 발화행위(locutionary act): 발화와 관련된 말하기 행위
b. 수행행위(illocutionaryact): 발화 안에 포함된 화자의 의도, 말의 힘
c. 결과행위(perlocutionary act): 발화 이후 나타나는 특정한 행위

(1)의 세 요소는 각각 분리된 행위가 아니라 소통의 상황에서 동시에 일어나는 행위이며, 이 중에서 가장 중심적인 위치를 차지하는 것은 수행행위이다. 수행행위는 발화를 통해 화자가 원하는 행동이 무엇인가를 나타내는 부분이고, 화자의 의도가 드러나는 부분이기 때문이다.

Austin(1962)에서는 판정행위(Verdicitives), 실행행위(Exercitives), 언약행위(Commissives), 행동행위(Behavitives), 설명행위(Expositives)의 5가지 유형으로 화행을 분류하였고[9], 이후 Searle(1976)에서 좀 더 구체적으로 확장되었다[11]. Searle(1976)은 화행을 동사의 유형이 아니라 행위로서 분류해야 한다는 입장에서 단언행위(Assertives), 지시행위(Directives), 언약행위(Commissives), 표출행위(Expressives), 선언행위(Declarations) 등 5가지로 나누고, 세부 유형으로 다음에 제시한 [표 1]과 같은 표현을 포함시키고 있다.

[표 1] Searle(1976)의 화행 유형

[Table 1] Types of Speech Act (Searle, 1976) 


\begin{tabular}{|c|c|c|}
\hline 화행의 유형 & 개념 & 세부 유형 \\
\hline 단언 행위 & $\begin{array}{l}\text { 화자가 자신이 믿는 내용을 언어적으로 표현하는 } \\
\text { 화행 }\end{array}$ & $\begin{array}{l}\text { 단언하기, 진술하기, } \\
\text { 결론맺기, 보고하기 }\end{array}$ \\
\hline 지시행위 & $\begin{array}{l}\text { 청자가 무엇인가를 하도록 하는 화자의 시도를 } \\
\text { 표현하는 화행 }\end{array}$ & $\begin{array}{l}\text { 충고하기, 명령하기, 주문하기, } \\
\text { 질문하기, 요청하기, 제안하기 }\end{array}$ \\
\hline 언약행위 & $\begin{array}{l}\text { 화자가 미래에 어떤 행위를 하겠다고 인식하게 } \\
\text { 하는 화행 }\end{array}$ & $\begin{array}{l}\text { 제공하기, 서약하기, } \\
\text { 거절하기, 위협하기 }\end{array}$ \\
\hline 표출행위 & 화자의 심리적 태도나 상태를 표현하는 화행 & $\begin{array}{l}\text { 사과하기, 비난하기, } \\
\text { 칭찬하기, 감사하기 }\end{array}$ \\
\hline 선언행위 & $\begin{array}{l}\text { 언어외적인 특정 제도 안에서 무엇인가를 인식하게 } \\
\text { 하는 화행 }\end{array}$ & $\begin{array}{l}\text { 선언하기, 선포하기, 제명하기, } \\
\text { 해고하기, 지명하기 }\end{array}$ \\
\hline
\end{tabular}

‘화행’은 특정한 상황이나 사실을 전달하는 발화를 넘어서서, 발화가 청자에게 어떤 영향을 미치는 행위이다. 또한 화자의 발화가 청자에게 영향을 미치기 위해서는 언어가 사용되는 사회적 맥락이나 상황에 대한 인식이 이루어져야 하는데, 이 부분은 화자의 의사소통 능력을 결정하는 중요한 요인이 된다.

\section{2 화행과 언어 사용}

언어 사용은 사회문화적 규범과 밀접하게 관련되어 있다. 따라서 한 언어를 익히기 위해서는 그 사회의 문화적 맥락을 익히는 것이 필요하다. 언어는 고정되어 있는 것이 아니라 발화 상황에 따라 화자가 어떤 의도를 가지고, 또 청자가 그 의도를 어떻게 이해하느냐에 따라 각기 다르게 해석될 수 있기 때문이다. 사회문화적인 배경을 바탕으로 이루어지는 의사소통 능력이란 특정 언어공동체 안에서 적절하게 의사소통을 하기 위해서는 무엇을 알아야 하며, 또 그렇게 하는 것을 어떻게 배우는가 하는 것이다. 또한 그러한 지식 및 그 지식을 활용하는 데에 필요한 모든 기술, 특히 의사소통의 규칙과 상호작용에 대하여 공유된 규칙뿐만 아니라 의사소통의 사례 및 상호작용 과정의 맥락과 내용의 기반이 되는 문화적인 규칙과 지식도 포함된다[12]. 따라서 이민자의 사회 적응과 의사소통 능력을 향상하기 위해서는 문화적 화행에 관한 교육이 이루어져야 한다. 문화적 화행의 대표적인 유형으로는 칭찬하기, 감사하기, 사과하기, 거절하기 등이 있다. 이와 같은 유형의 화행은 일상적인 대화 상황에서 빈번하게 사용될 뿐만 아니라 각기 다른 사회문화적인 차이를 나타내기도 한다. 특히 감사와 사과 화행은 화자 자신의 심리적 상태를 나타내는 '표출 화행'이어서 화자와 청자 사이에 감정적 공감이 이루어져야 하는데, 사회문화적 공감대가 없다면 의도하지 않은 오해가 생길 수도 있어 주의가 필요한 화행이다.

\section{3. 사회통합 프로그램에서의 의사소통 기능}

\section{1 사회통합 프로그램의 특성과 교육과정}

법무부에서 주관하는 사회통합 프로그램은 한국에 체류하는 이민자가 우리 사회에 
안정적으로 정착하여 건전한 사회 구성원으로 자립하는 데에 필요한 기본소양(한국어와 한국문화, 한국사회 이해)을 체계적으로 습득할 수 있도록 마련한 교육 프로그램이다. 교육을 이수한 이민자에게는 체류 허가를 부여하고 영주권 또는 국적을 부여하여 이민정책과 연계한 혜택을 제공함으로써 참여자의 성취도를 높이고 교육효과를 극대화하여 사회통합 정책의 핵심적인 역할을 수행하는 것이 프로그램의 목표이다[13].

사회통합 프로그램의 정규 교육과정은 총 5 단계로 구성되어, 한국어와 한국문화에 대한 교육 최대 415시간, 한국사회 이해에 대한 교육 70 시간으로 편성되어 있으며, 전체적인 교육과정은 다음의 [표 2]와 같다.

[표 2] 사회통합 프로그램 과정 및 이수 시간

[Table 2] Social Integration Program Course and Completion Hours

\begin{tabular}{|c|c|c|c|c|c|c|c|}
\hline 구분 & \multicolumn{5}{|c|}{ 한국어와 한국문화 } & \multicolumn{2}{|c|}{ 한국사회 이해 } \\
\hline 단계 & 0 단계 & 1단계 & 2단계 & 3단계 & 4단계 & \multicolumn{2}{|c|}{ 5단계 } \\
\hline 과정 & 기초 & 초급1 & 초급2 & 중급1 & 중급2 & 기본 & 심화 \\
\hline 이수시간 & 15 시간 & 100시간 & 100 시간 & 100 시간 & 100시간 & 50시간 & 50시간 \\
\hline 평가 & 없음 & 1단계평가 & 2단계평가 & 3 단계평가 & 중간평가 & 영주용종합평가 & 귀화용종합평가 \\
\hline 참고 & & $\begin{array}{l}\circ 5 \text { 단계 } \\
※ \text { 영주용 }\end{array}$ & $\begin{array}{l}\text { l화과정은 } \\
\text { 종합평가 }\end{array}$ & $\begin{array}{l}\text { 본과정 }= \\
\text { 작자는 기 }\end{array}$ & (수료인) & $\begin{array}{l}\text { 석시간 수강) } \\
\text { 읺고 심화과 }\end{array}$ & $\begin{array}{l}\text { 참여 } \\
\text { 참여 }\end{array}$ \\
\hline
\end{tabular}

한국어 교육은 기초, 초급 1 , 초급 2 , 중급 1 , 중급 2 의 5 개 과정으로 나누어 학습자들이 자신의 수준에 맞는 단계부터 순차적으로 배울 수 있도록 구성되어 있다. 기초 단계는 한국어 자모 학습부터 시작하는 단계이다. 초급의 1 단계는 기초적 단어와 문장을 학습하여 기본적인 의사소통 능력을 기르고, 2 단계는 제한된 수준이지만 어휘와 문단을 활용하여 어느 정도 한국어를 구사 할 수 있는 단게이다. 중급의 1,2 단계에서는 큰 어려움 없이 원어민과의 일상적인 의사소통이 가능하도록 한국어를 어느 정도 구사할 수 있도록 하는 단계이다. 정규과정 이후, 고급 단계에서는 원어민과 의사소통을 원활하게 하며 자신의 의사표현과 일상적인 업무수행을 어려움 없이 수행할 수 있도록 하는 것을 목표로 한다[13].

사회통합프로그램에 참여하는 학습자가 한국 사회에 완전히 통합되는 단계까지 도달하기에는 많은 시간이 필요하겠지만, 이 프로그램은 이민자가 새로운 한국 사회와 문화에 적응할 수 있게 할 뿐만 아니라 의사소통의 참여자로서 충분한 역할을 할 수 있도록 성장하고 발전할 수 있게 한다.

\section{2 사회통합 프로그램 한국어 교재 분석}

사회통합 프로그램은 국가가 정책적으로 기획하여 운용하고 있는 프로그램이어서 교육 목적과 방향은 분명하지만 교육 내용의 실제성이나 상호문화주의적인 관점의 문화 교육과 다양한 의사소통 상황을 충분히 담지 못한 부분에 대한 지적이 있었다[1]. 그럼에도 불구하고 사회통합 프로그램에서 활용되는 한국어 교재는 국적 취득을 목적으로 하는 이민자가 한국어 의사소통 능력을 향상할 수 있게 하는 중요한 교재이며, 
한국 사회와 문화에 적응할 수 있도록 도와줄 수 있는 필수적인 요소이다.

신규 개발 교재에서 가장 중점을 두고 있는 부분은 이민자들의 한국 생활과 관계 깊은 주제, 어휘와 표현, 상황, 텍스트, 과제 등을 선정함으로써 살아 있는 언어문화 교육이 되도록 하는 것으로, 교재 발간에 관한 결과 보고서에서 밝힌 각 단계별 교육목표는 다음의 [표 3]과 같다[8].

[표 3] 사회통합 프로그램 교육과정의 단계별 총괄 목표

[Table 3] Overall Goals for Each Stage of the Social Integration Program Curriculum

\begin{tabular}{|c|c|}
\hline 단계 & 목표 \\
\hline $\begin{array}{l}\text { 기초단계 } \\
\text { (0단계) }\end{array}$ & $\begin{array}{l}\text { 한글 자음 모음을 익혀 간단한 기초 어휘를 읽고 쓸 수 있다. 한국어의 기본 문장을 한국인 } \\
\text { 원어민 화자가 이해할 수 있을 정도로 발음할 수 있다. }\end{array}$ \\
\hline $\begin{array}{l}1 \text { 단계 } \\
\text { (초급1) }\end{array}$ & $\begin{array}{l}\text { 이주민이 한국 생활에서 자주 접하는 개인적인 상황에서 기초적인 어휘, 정형화된 표현을 } \\
\text { 사용하여 의사소통을 할 수 있다. 또한 가장 기본적인 한국의 일상생활 문화를 이해하고 } \\
\text { 적용할 수 있다. }\end{array}$ \\
\hline $\begin{array}{l}2 \text { 단계 } \\
\text { (초급2) }\end{array}$ & $\begin{array}{l}\text { 일상생활과 관련된 주제로 간단하게 의사소통을 할 수 있으며, 한국생활에서 자주 접하는 } \\
\text { 공공장소(우체국, 은행 등)에서 간단한 과제를 수행할 수 있다. 한국 생활에 관련된 일상생활 } \\
\text { 문화를 이해하고 적용할 수 있다. }\end{array}$ \\
\hline $\begin{array}{l}3 \text { 단계 } \\
\text { (중급1) }\end{array}$ & 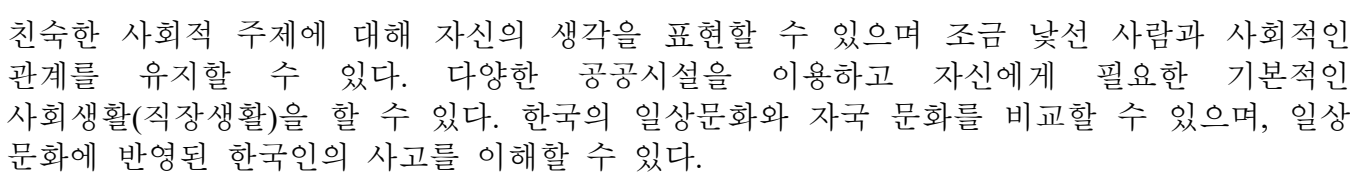 \\
\hline $\begin{array}{l}4 \text { 단계 } \\
\text { (중급2) }\end{array}$ & $\begin{array}{l}\text { 일반적인 사회적 주제에 대해 자신의 의견을 말할 수 있으며, 대상과 상황에 맞게 격식과 } \\
\text { 비격식을 사용할 수 있다. 자신과 밀접하게 관계된 사회적 업무를 수행할 수 있다. 한국의 } \\
\text { 사회문화적 특징을 이해할 수 있으며, 한국 사회 일원으로 기본적인 법과 질서를 지킬 수 } \\
\text { 있다. }\end{array}$ \\
\hline
\end{tabular}

위에 제시된 목표에 따라 신규 개발된 교재는 모두 5 단계로 나누어져 있다. 새 교재는 15 시간 분량의 4 개 단원으로된 기초 단계, 각각 총 100 시간 분량의 20 개 단원으로 구성된 초급 1,2 단계와 18 개 단원으로 구성된 중급 1,2 단계로 구성된, 언어와 문화가 통합된 교재이다. 교재의 교수요목은 주제, 기능, 문법, 문화와 정보, 네 가지 요소로 구성되었다. 그러면 다음 절에서는 자모를 학습하는 기초단계를 제외하고, 1 단계부터 4단계 교재의 교수 요목 중에서 주제와 기능에 대한 분석 결과에 대해 제시하고자 한다.

\subsection{1 교재의 주제에 대한 분석 결과}

교재에서 선정한 주제는 국제통용 한국어 표준 모형(이하 표준모형, 2017)을 반영하여, 초급 1 단계에서는 인사, 교통, 음식 등 기초 생활과 관련된 것과 '한국생활'이라는 주제를 별도로 구성하여 입국 전 한국인의 생활에 대해 이해할 수 있도록 하였으며, 초급 2단계에서는 자신에 대한 세부 묘사와 공적 업무 수행에 필요한 주제를 선정하였다. 또한 중급 1 단계에서는 공적관계와 공적 업무를 주제로 구성하고, 대인관계에서의 예절을 다루고, 한국 문화를 좀 더 깊이 이해하도록 구성하였으며, 부동산에 대해 이해하고 과제를 수행하도록 하였다. 또한 한국의 이민 생활에서 직장 생활과 취업 활동에 대한 요구가 높다는 점을 반영하여 직장 생활에서의 다양한 업무와 구직 활동에 대한 준비 등의 내용 등을 도입하였다. 중급 2 단계에서는 한국의 법과 질서, 정책 문제 등의 주제를 통해 한국 사회를 이해하도록 함은 물론 국제 사회와 다문화 사회의 특징을 
알고 그 구성원으로서 살아가는 데에 필요한 것이 무엇인지 익히도록 주제를 구성하였다[8].

기존 교재와 달리 새로 개발된 교재는 이민자의 특성을 고려하여 주제를 선정한 노력이 보인다. 초급 1 단계에서는 '한국생활'이라는 단원을 별도로 구성하여 한국 생활에 필요한 정보를 확인할 수 있도록 한 점, 초급 2단계에서는 우체국과 은행, 출입국이나 외국인청 등 공공기관을 이용하는 데에 활용할 수 있는 주제를 선정한 점, 중급 1 단계에서는 구직 활동, 한국의 직장생활 등의 주제를 선정하여 사회 구성원으로서의 역할 수행에 필요한 내용을 다루고, 중급 2단계에서 이주민의 정착에 초점을 맞춘 주제를 선정한 점 등이 이주민의 실제 생활과 연결되어 있는 주제를 선정했다는 점에서 돋보이는 부분이다. 특히 기존 교재의 초급에서는 기초적인 의사소통을 중심으로 주제가 선정되었으나 새 교재에서는 이민자들이 이미 한국 사회에 노출되어 있는 점을 고려하여 초급 단계부터 이민자의 상황에 대한 부분을 주제로 포함한 것도 긍정적이라고 하겠다. 또한 중급 1 단계에서는 '대인관계'를 주제로 선정하고 대인관계의 어려움이나 관계 회복에 대한 내용을 다루고 있는데, 이는 동일한 사회 구성원으로서 다른 사람들과의 인간관계를 유지하는 것이 사회생활의 가장 기본이 되는 요소라는 점을 강조한 것이어서 매우 유용하고 적절한 접근이라고 볼 수 있다.

\subsection{2 교재의 기능에 대한 분석 결과}

교육과정의 구성요소 중에서 화행 사용 양상을 살펴볼 수 있는 부분은 '기능' 부분이다. 한국어 교육에서 '기능'은, 언어 형태를 기반으로 의사소통을 수행하는 것으로 정의하고 있기 때문에 화행과 가장 밀접하게 연결할 수 있는 요소이다.

교재 개발 결과 보고서에 따르면, 초급 1 단계에서는 개인적인 주제 범주 안에서 생존 기능과 같은 제일차적인 기능을 중심으로 구성하여, 자기자신, 가족, 친구를 소개하거나 기본적인 동사와 형용사로 자신을 말하고, 기초 생활을 영위하는 데에 필요한 기능을 익히도록 하였다. 초급 2 단계에서는 '기본적인 한국 생활'이라는 단계에 머무르지 않고 이민자가 한국의 공공시설을 이용하거나 문화생활을 즐기면 살아갈 수 있는 기능을 익히도록 하였다[8].

표준 모형에서는 양적, 질적으로 방대해지는 한국어 교육의 형식적-내용적 측면의 발전을 위해, 객관적 준거로서 기능할 수 있는 교육과정의 설계와 수립에 관한 기준을 제안하고 있는데, 이 모형에서는 한국어 교육에서 학습해야 할 기능을 90개로 제시하고 있다[14]. 그중에서 초급과 중급에서 선정된 기능을 기준으로 새 교재에서 선정한 기능과 비교해서 살펴보면 다음의 [표 4]와 같다.

[표 4] 선정된 기능 비교 분석

[Table 4] Comparative Analysis of Selected Functions

\begin{tabular}{|c|c|c|}
\hline 구분 & 국제통용 한국어 표준 모형 & 분석 교재 \\
\hline 초급 1 단계 & $\begin{array}{l}\text { 묻고 답하기, 약속하기, 계획하기, 문의하기, } \\
\text { 제안하기, 주문하기, 조언하기, 인사하기, 흥 } \\
\text { 정하기, 부탁하기 }\end{array}$ & $\begin{array}{l}\text { 묻고 답하기, 약속하기, 계획하기, 주문하기, 인사 } \\
\text { 하기, 질문에 답하기, 소개하기, 조언하기, 서술하 } \\
\text { 기, 정보전달하기 }\end{array}$ \\
\hline 초급 2 단계 & $\begin{array}{l}\text { 묻고 답하기, 제안하기, 정보구하기, 문의하 } \\
\text { 기, 조언하기, 칭찬하기, 금지하기, 거절하기, } \\
\text { 설명하기, 약속하기, 격려하기, 비교하기, 걱 } \\
\text { 정하기, 부탁하기, 감정표현하기 }\end{array}$ & $\begin{array}{l}\text { 소개하기, 부탁하기, 물건사기, 전화하기, 조언하 } \\
\text { 기, 초대하기, 설명하기, 추측하기, 경험 말하기, } \\
\text { 정보전달하기 }\end{array}$ \\
\hline
\end{tabular}




\begin{tabular}{|c|l|l|}
\hline \multirow{2}{*}{ 중급1 단계 } & $\begin{array}{l}\text { 묻고 답하기, 조언하기, 제안하기, 문의하기, } \\
\text { 소개하기, 정보구하기, 후회표현하기, 설명하 } \\
\text { 기, 사과하기, 전구하기, 소개하기, 묻고 답하기, 경험 말하기, 추천하기, 계획하기, } \\
\text { 부탁하기, 약속하기 }\end{array}$ & $\begin{array}{l}\text { 조언하기, 소감 말하기, 문의하기, 비교하여 말하 } \\
\text { 기보 전달하기, 고민 말하기 }\end{array}$ \\
\hline 중급2 단계 & $\begin{array}{l}\text { 묻고 답하기, 설명하기, 주장하기, 격려하기, } \\
\text { 제안하기, 추천하기, 정보구하기, 걱정하기 }\end{array}$ & $\begin{array}{l}\text { 계획하기, 절차에 따라 말하기, 소개하기, 조언하 } \\
\text { 깅험 말하기, 찬반 의견 말하기, 제안하기 }\end{array}$ \\
\hline
\end{tabular}

[표 4]에서와 같이, 교재에 나타난 의사소통 상황의 기능은 표준 모형에서 제시하고 있는 기능과 같은 단계의 기능도 있지만 그 수가 많이 줄어든 것을 볼 수 있다. 또한 표준 모형에서는 중급 1 단계에서만 다루는 '소개하기'가 초급 1 단계부터 중급 2 단계까지 반복적으로 제시되고 있어 이민자의 위치나 역할을 고정한 것처럼 여겨진다. 또한 대화를 이어가는 데에 가장 기본이 되는 '묻고 답하기'라는 대응쌍은 초급과 중급 1 단계에서만 각각 선정되어 있다. 그러나 대화를 구성하는 기본적인 기능에 관한 연습을 강조하기 위해서는 표준 모형과 같이 모든 단계에서 그 난이도를 달리하여, 대화가 이어져 나가는 방법을 익혀야 할 것이다. 더불어 이주민이 만날 수 있는 여러 상황에서 활용할 수 있도록 좀 더 다양한 기능이 포함되도록 그 수를 확장할 필요가 있다. 물론 표준 모형은 한 단계의 시수가 200시간이고, 사회통합 프로그램은 100 시간을 기준으로 구성되어 있기 때문에 단순히 비교하기에는 한계점이 있다.

그런데 무엇보다도 가장 두드러진 부분은 새교재에서는 표준 모형에서 제시된 칭찬하기, 사과하기, 거절하기, 감사하기 등에 해당하는 기능이 다루어지지 않아 이주민의 한국 사회 적응에 필요한 실제적 기능을 학습하기 어려운 부분이 있다는 것이다. 문화적 화행이라고도 불리는 이들 화행은 일상적으로 사용되면서 인간관계의 기본이 되지만, 활용하는 방법에 있어서는 문화적으로 차이가 나타나기 때문에 반드시 학습이 필요하다. 사과 화행은 수행하는 화자의 입장에서는 자신의 체면이 상할 수도 있지만 그 대상이 되는 청자의 입장에서는 체면을 인정해 주는 행위라는 점에서 공손의 한 형태가 된다. 물론 지나친 사과는 오히려 상대방을 불편하게 할 수도 있지만 이주민의 경우 의도하지 않은 실수나 오해가 많을 수 있기 때문에 자신의 행위에 대해 상대방의 이해를 구하는 공손함이 필요한 경우에 유용하게 활용할 수 있는 화행이다. 거절 화행은 상대방의 요청이나 제안, 약속 등을 수용하지 않고 거부의 의도를 나타내는 화행이다. 거절은 요청, 제안, 약속, 초대 등에 대한 비선호적인 받는 말이기 때문에 잘못 수행하는 경우에는 상대방과 갈등을 일으킬 수도 있는 화행이다. Coulmas(1981)에서는 감사 화행의 적절한 사용 규칙은 각 문화권마다 다르기 때문에 외국인 학습자가 오해나 어려움을 겪게 되는 부분이라는 것을 지적하고 있는데[15], 이와 같은 연구 결과는 이주민을 위한 언어 교육에서 화행 사용의 양상에 대한 교육이 반드시 이루어져야 한다는 것을 확인하게 한다.

이주민의 사회적응 교육에 언어 교육이 포함되는 것은 단순히 그 언어의 문법에 맞도록 어휘를 사용한다는 것만이 아니라 의사소통 능력을 향상한다는 목적을 달성하기 위해서일 것이다. 문화적 화행은 인간관계를 이어주는 예절의 관점에서 중요한 역할을 하며, 의사소통 능력의 향상이라는 목표 달성을 위한 필수적인 화행이다. 새로 개발된 교재에서 이주민의 한국 사회 적응에 도움이 될만한 주제에 대해서 대해 다루고 있는 점은 매우 긍정적이지만 이를 뒷받침할 실제적 기능과 문화적 화행에 대한 제시가 부족하다는 점은 아쉬운 부분이다. 따라서 긍정적인 인간관계를 유지하기 위해서는 그 
사회에서 통용되는 화행 사용의 방식을 이해하고, 거기에 맞추어 수행할 수 있는 보완책을 마련해야 할 것이다.

\section{4. 결론}

이 연구에서는 사회통합프로그램의 한국어 교재 발간에 관한 연구 보고서 내용을 토대로 2020년에 새로 발간된 교재의 주제와 기능을 화행의 관점에서 확인하고, 이민자들의 원활한 의사소통에 필요한 교육 내용을 포함하고 있는지 확인하였다.

분석 결과, 초급과 중급 교재는 모두 학습 시간에 맞추어 체계적으로 구성되어 있으며, 주제면에 있어서 이전의 교재와는 달리 이주민의 상황을 최대한 반영하기 위한 노력이 돋보였다. 그러나 기능적인 면에 있어서는 기준으로 삼은 표준모형보다도 제시된 기능의 수가 적었고, 무엇보다도 문화적 화행에 대해 다루지 않은 점이 매우 아쉬운 부분이다. 의사소통의 측면에서 이주민의 의사소통 능력 향상을 위한 화행 교육 내용에 대한 전면적인 검토가 필요하며, 사회통합 프로그램에서 다루는 기능으로 칭찬하기, 사과하기, 거절하기, 감사하기 등의 문화적 화행을 폭넓게 다루어야 한다. 이는 이민자가 한국 문화를 이해하고 이를 토대로 우리 사회의 구성원으로서 적응하고 자립할 수 있는 더 나은 방안을 마련하기 위해 필수적인 요소이다. 또한 교재의 부족한 기능에 대한 활용은 보조 자료와 실제적 활동을 통해 충분히 보완될 수 있도록 해야 할 것이다.

이 연구는 교재의 대화 상황을 하나하나 살펴보지는 않은 점이 한계로 지적될 수 있으나 교재 발간에 관한 결과 보고서를 중심으로 분석했기 때문에 사회통합 프로그램 한국어 교재의 내용과 의도를 충분히 확인했다고 할 수 있다. 또한 이 연구의 결과는 이민자가 사회통합 프로그램의 한국어 교재를 통해 문화적 차이를 극복하고, 의사소통 능력을 향상하는 데에 조금 더 기여할 수 있는 바탕이 될 것이다.

\section{5. 감사의 글}

이 논문은 2021 년 상명대학교 교내연구비 지원을 받은 연구입니다.

\section{References}

[1] Jung-mi Jeon, Analysis of Intercultural education textbook in Immigration \& Integration Program of Korea, Korean Journal of culture and arts education studies, (2019), Vol.14, No.1, pp.83-113.

[2] Hang-rok Cho, A Study on Extending Implement of Korean Language Education in KIIP (Korea Immigration Integration Program), Bilingual Research, (2012), No.50, pp.235-267.

[3] Ho Seon Jeong, A Study on the Development of Korean Language Textbooks for Social Integration of Immigrants in Korea, Doctorial Dissertation, Sang Myung Univerersity, (2016)

[4] Young Yun, Kyeong-Ae Son, A Study on the Contents and Methods of the Korean Cultural Education for the Social Integration of Immigrants, The Language and Culture, (2016), Vol.12, No.3, pp.123-154.

[5] Rae-ho Lee, Analysis of the Organization and Contents of Korean Textbook for Korea Immigration \& Integration Program [KIIP], The Journal of Yeongju Language \& Literature, (2015), Vol.29, pp.347-374.

[6] Mihye Lee, Current Position and Direction of Development of Korean Language Curriculum for Social Integration Program, The Fifth Internaional Conference proceedings on Korean Language Education, (2015), August 8-9; Seoul, Korea. 
[7] Mihyang Lee, Young Yun, Eunji Choi, Jinwook Park, A Study on Improving the Korea Immigration \& Integration Program's Korean Curriculum and Textbook for Immigrants as a Specific Purposes, Journal of the International Network for Korean Language and Culture, (2018), Vol.15, No.1, pp.147-174.

[8] Mihye Lee, The Development of Korean Language teaching materials for Korea Immigration \& Integration Program in 2019, Korea: National institute of Korean Language, (2019)

[9] J. L. Austin, How to do things with words, UK: Oxford University Press, (1962/1975)

[10] J. R. Searle, Speech acts: An essay in the philosophy of language, UK: Cambridge University Press, (1969)

[11] J. R. Searle, A classification of illocutionary acts, Language in Society, (1976), Vol.5, No.1, pp.1-23.

[12] M. Saville-Troike, The ethnography of communication: An introduction, UK Oxford: Blackwell Publishing, (2003)

[13] https://www.socinet.go.kr/soci/contents/PgmCosCopl.jsp?q_global_menu_id=S_SIP_SUB02, Mar 23 (2021)

[14] Jung Seob Kim, The Internationally Used Korean Education Standard Model 2, Korea: National Institute of Korean Language, (2011)

[15] F. Coulmas, Poison to your soul: Thanks and apologies contrastively viewed, Conversational routine: Explorations in standardized communication situations and patterned speech, German: De Gruyter Mouton (1981). 